\title{
Self-stabilizing Tiny Interaction Protocols
}

\author{
Davide Canepa Maria Gradinariu Potop-Butucaru \\ Université Pierre et Marie Curie (Paris 6), CNRS,INRIA, France \\ canepa.davide@tiscali.it, maria.gradinariu@lip6.fr
}

\begin{abstract}
In this paper we present the self-stabilizing implementation of a class of token based algorithms. In the current work we only consider interactions between weak nodes. They are uniform, they do not have unique identifiers, are static and their interactions are restricted to a subset of nodes called neighbours. While interacting, a pair of neighbouring nodes may create mobile agents (that materialize in the current work the token abstraction) that perform traversals of the network and accelerate the system stabilization. In this work we only explore the power of oblivious stateless agents. Our work shows that the agent paradigm is an elegant distributed tool for achieving selfstabilization in Tiny Interaction Protocols (TIP). Nevertheless, in order to reach the full power of classical self-stabilizing algorithms more complex classes of agents have to be considered (e.g. agents with memory, identifiers or communication skills). Interestingly, our work proposes for the first time a model that unifies the recent studies in mobile robots(agents) that evolve in a discrete space and the already established population protocols paradigm.
\end{abstract}

\section{Introduction}

Recently, the distributed computing community started to investigate the interactions in biological and chemical systems in order to provide efficient computational models for adhoc systems like sensor or peer-to-peer. One of the most promising research in this direction is the population protocol model developed by Angluin et al. through a series of papers [1, 2]. In this model, pairs of nodes chosen by an adversary interact and change their state according to a transition function. In [1] it is stated that for each such transition function, the resulting population protocol is said to stably compute a predicate on the initial states of the nodes if, after sufficiently many interactions in a fair execution, all nodes converge to having the correct value of the predicate. Nodes in this framework have no identity and no ability to distinguish two different interactions with the same node. Interactions between nodes in this model have various incarnations. Sometime interactions are restricted by the choice of a fair or randomized scheduler while in other situations the network topology is the main parameter that defines them. This corresponds perfectly to the real networks. In sensor networks, for example, the topology (the geographical position of nodes) is the main parameter having a major impact on sensor interactions while in peer-to-peer networks the interactions are restricted to the virtual overlay defined by the peers.

The main concern was to evaluate the computational power of the population protocols model and several problems have been addressed: coloration, phase synchronization, counting, leader election [1, 3, 2]. Fault tolerance and security issues in variants of the model have been addressed in [4] 5]. Selfstabilizing population protocols have been further addressed. One of the first problems that has been investigated was leader election and the first work that addresses this issue [2]. Interestingly, the self-stabilizing extension of classical population protocols quickly meet dead-ends. Several impossibility results have been proved. Therefore, $[2 \mid$ extends the study to the non-uniform leader election in odd and directed rings. Furthermore, due to the persisting impossibility results the model is enriched with fairness assumption and oracles (abstractions that offer some global information). Fisher and Jiang study the self-stabilizing leader-election problem in this model in [6]. They introduce $\Omega$ ? an oracle that reports true or false if it detects the presence or the absence of a leader. Using $\Omega$ ?, the authors provide uniform and self-stabilizing leader election algorithms for fully connected networks under the assumption of local fairness and for rings under the global fairness assumption. In [6] the authors also prove that uniform leader election is impossible in rings assuming local fairness, even with the help of $\Omega$ ?. In the current work, we prove that this result holds even under global fairness without additional assumptions. In [7] the authors investigate the memory necessary to solve the problem without oracles.

Another problem addressed in the classical model of population protocols is the self-stabilizing token circulation. This problem has been addressed only in non-uniform population protocols [2]. Our work extends the study to the uniform pop- 
ulation protocols. We prove the possibility of deterministic solutions only on chains and using global fairness assumptions. For the general graphs we propose an impossibility result and a probabilistic solution.

These studies prove that the self-stabilization in population protocols model reached a dead-end when dealing with both static (e.g. leader election) or dynamic tasks (e.g. token circulation). Therefore, a recent variant of the model explores the self-stabilization of the system when the interactions benefit from the presence of a base station with incorruptible infinite memory. This model, extremely powerful by its hypothesis already proved its effectiveness since most of the tasks selfstabilizing in classical distributed settings found also a solution in this model [8]. However, the main challenge to address would be the minimum assumptions one has to make on the interaction system in order to reach its stabilization. One natural idea is the use of the popular paradigm of mobile agents. This paradigm already proved its efficiency in optimizing the self-stabilization in classical distributed settings [9, 10].

Interestingly, the "probes" used in the Fisher's leader election algorithm to "destroy" the other leaders in the population are very similar to the agents paradigm. Therefore, the extension of population protocols with mobile agents seems a promising research direction. Our goal is to unify the population protocols model and the agents paradigm. We advocate that the computational power of the population protocols can benefit from the agents ability to "move" from one node to another and hence to disseminate or gather information. The model becomes even more interesting when agents are allowed to execute some code when they are guested by a particular node.

\subsection{Our contribution}

We propose a novel model of interactions that extends the population protocols with the power of agents and oracles schemes. In the current work we explore the self-stabilization power of the weakest version of this model, TIP (Tiny Interaction Protocols): the interactions between nodes is restricted to a predefined neighbourhood, nodes and agents are oblivious, anonymous and uniform. In this model we consider the self-stabilization of both dynamic and static tasks. More specifically, we consider two popular case studies in selfstabilization: the token circulation and the leader election (in its local and global version).

Our contribution is threefold. First, we propose some negative results related to the feasibility of leader election and token circulation in the TIP model. Then, we present some positive results: self-stabilizing solutions for token circulation in chains and arbitrary graphs. Furthermore, we propose solutions for self-stabilizing local leader election. Finally, we prove for both static and dynamic tasks the necessity of an oracle that provides to each node information about the current state of the system. Interestingly, we prove that in the case of the token circulation this information has to be global in both deterministic and probabilistic settings while for the case of the local leader election only a local knowledge is sufficient. We also show that in the case of the global leader election even the global knowledge is not sufficient to solve the problem. In this case, additional assumptions (e.g. memory on agents or nodes) have to be made.

\subsection{Paper Road-map}

The paper is organized as follows. Section 2 proposes de TIP model. In Section 3.1 we provide some impossibility results related to the token circulation in TIC. Sections 4 proposes deterministic and probabilistic solutions for token circulation in some particular classes of graphs. In Section 5 we address the local leader election and propose a negative result for the global leader election even with the help of the global fairness.

\section{Model}

\subsection{Interaction Protocols with mobile agents}

We represent a network by a fixed undirected graph $G=$ $(V, E)$. Each vertex represents a finite-state sensing device and an edge $(u, v)$ indicates the possibility of interaction between $u$ and $v$ in which $u$ is the initiator and $v$ is the responder. For a node $u, \mathcal{N}_{u}$ is the set of all the nodes $v_{i}$ responder of $u$.

Each node may have a local set of variables and a memory slot reserved for storing one or several agents. An agent is a mobile abstraction (e.g. message, mobile program) that can be locally created, deleted, modified, executed or sent by a node to another node. Each node can access a local primitive, LocalAgent?() which returns true if locally there is an agent. When interacting with another node, a node can create a local agent, destroy the local agent, execute the local agent or push the local agent to the peer node in the interaction.

A local state of a node is given by the value of its local variables and the state of the local agent if LocalAgent?() invocation returns true. The system can be modelled as a transition system. When two nodes $u$ and $v$ interact, their state changes atomically from $\left(s_{u}, s_{v}\right) \rightarrow\left(s_{u}^{\prime}, s_{v}^{\prime}\right)$, referred in the following as local transition. A local transition may be either deterministic or probabilistic. A configuration of the system at some time $t$ is the set of states of the nodes in the system at $t$. Contrary to existing models for population protocols our model allows several pair of nodes to interact in a given configuration. The only restriction we impose is that concurrent interactions are pairwise independent. That is, two pairs of nodes that interact at the same time $t$ have no common node. Let $c_{t}$ be the configuration of the system at time $t$. There is a global transition of the system at time $t$ from $c_{t}$ to $c_{t+1}$ if there is at least one local transition in $c_{t}$. An execution of the system, $e$, is a sequence of configurations $e=\left(c_{1}, c_{2}, \ldots\right)$ where $c_{i+1}$ is obtained from $c_{i}$ by a global transition.

\subsection{Schedulers and Fairness}

Intuitively, a scheduler in population protocols chooses the pairs of agents that will interact in a given configuration. Formally a scheduler is a predicate over the executions of the system. In this paper we consider the weakly fair version of the following schedulers : arbitrary and k-bounded. A scheduler is 
weakly fair if, in an infinite execution, a continuously enabled pair of agents is eventually activated.

- $k$-bounded: between two consecutive activations of a pair of agents, another pair of nodes can be activated at most $k$ times;

- arbitrary: at each configuration an arbitrary subset of pair of nodes is activated.

Note that contrary to some work done in population protocols the above schedulers are not randomized. A randomized scheduler is one of the weakest schedulers one may consider therefore this type of schedulers are not the object of the current work.

We also consider a stronger version of the above defined weak fairness referred in the population protocols literature as global fairness. With global fairness an interaction that is infinitely often possible in an execution is infinity often scheduled.

\subsection{Faults and Self-stabilization}

In this paper we assume that nodes can start their execution in any configuration. For the particular case of token circulation or leader election the faulty period may cause the lost or the creation of the agent that materializes the token and nodes have no possibility to detect locally this faulty state. In order to deal with this kind of faults we use oracles and self-stabilization tools.

A self-stabilizing system [11] started in an arbitrary configuration eventually exhibits a correct behaviour according to its specification.

Definition 1 (SELF-STABILIZATIONS). Let $\mathcal{L}_{\mathcal{A}}$ be a nonempty legitimacy predicate (legitimacy predicate is defined over the configurations of a system and is an indicator of its correct behaviour) of an algorithm $\mathcal{A}$ with respect to a specification predicate Spec such that every configuration satisfying $\mathcal{L}_{\mathcal{A}}$ satisfies Spec. Module $\mathcal{A}$ is self-stabilizing with respect to Spec iff the following two conditions hold:

(i) Every computation of $\mathcal{A}$ starting from a configuration satisfying $\mathcal{L}_{\mathcal{A}}$ preserves $\mathcal{L}_{\mathcal{A}}$ (closure).

(ii) Every computation of $\mathcal{A}$ starting from an arbitrary configuration contains a configuration that satisfies $\mathcal{L}_{\mathcal{A}}$ (convergence).

The merge between population protocols and failure detectors was made for the first time in [6] where an eventual leader oracle (eventual leader detector), $\Omega$ ? is introduced to solve leader election. This oracle is useful when the system is started in symmetric configurations (no leader is elected). Note that the eventual leader detector $\Omega$ ? is a weaker version of the oracle $\Omega$ introduced first in [12] and proved to be the weakest failure detector to solve consensus. Instead of electing a leader (as $\Omega$ does), $\Omega$ ? reports to each node whether or not at least one leader is present in the network. Note that the guess may be correct or not and different guesses may be reported to different nodes. The only guarantee offered is that from some point onward if there is continuously a leader or if there is contin- uously no leader, $\Omega$ ? eventually accurately reports this fact to all nodes.

In this paper, we will use the eventual agent detector. Similar to the eventual leader detector defined in [6], the agent detector reports if at least one agent is present in the network.

DEFINITION 2 (EVEnTUAL AGENT DETECTOR). The eventual agent detector, A? supplies a Boolean input to each node at each step so that the following conditions are satisfied by every execution $e$ :

- If all but finitely many configurations of e lack of agent, then each node receives input false at all but finitely many steps.

- If all but finitely many configurations of e contain one or more agents, then each node receives input true at all but finitely many steps.

In mobile robots, [13], a similar abstraction is used: the unlimited robots visibility. The originality of our approach is to address the geographical power of the oracle. Interestingly, for some tasks it is sufficient that the oracle provides only a local information (e.g. for the case of the local leader election it is sufficient only the one hop distance information as discussed later in the paper). In the case of the global leader election we prove that this problem is impossible to solve even if this oracle offers information on the whole network.

\subsection{Leader election and Unique Token cir- culation}

In this paper we address two well known problems in distributed computing : leader election and token circulation. These two problems are similar in the sense that they share the safety property : a unique token/leader should be present in the system in any configuration (a token is a predicate over the local configurations of a node). However, the liveness part is different. In the leader election the unique token should be hold by the same node and no other node in the system should hold the token in the subsequent configurations while in the token circulation the unique token has to perpetually visit every node in the system. In our study, the token will be materialized by an agent.

DEFINITION 3 (UNIQUE TOKEN LEGITIMATE CONFIGURATION). A configuration is legitimate for the unique token iff exactly one node holds the token in this configuration.

DEFINITION 4 (UNIQUE TOKEN CIRCUlation). A system is self-stabilizing for the token circulation specification iff (i) each execution of the system converges in a finite number of steps to a unique token legitimate configuration and (ii) each process in the system holds the unique token infinitely often.

Definition 5 (SILENT LeAder Election). A system is silently self-stabilizing for the leader election specification iff each execution of the system converges in a finite number of steps to a unique token legitimate configuration and no node is enabled in that configuration. 
In this paper we also address the local leader election which restricts the election to a neighbourhood. That is, each process has to have a unique leader in its neighbourhood. Note that the local leader election is a weaker version of the MIS problem which focus on optimizing the set of local elected leaders to the minimal set. Recent local algorithms that addressed this problem are proposed in [14]. Interestingly the transformation of these algorithms in the population protocols model has not been explored yet.

\subsection{Work hypothesis}

In the current paper we assume a network of small devices with a static topology. Nodes do not have unique identification. The interactions between nodes follow the interactions model described above and are restricted by the topology of the network and the scheduler choice. During their interactions nodes can create agents that may further change their locations. A node invokes the LocalAgent?() primitive in order to detect the local presence of the agent. In the following we assume the weakest class of agents: anonymous and memoryless. That is, the agents do not carry any memory or code to be executed by their hosts. The only operations nodes can execute during an interaction: check if they hold locally an agent, create an agent or delete/push the local agent to the peer node. We also assume that each node receives Boolean inputs from the eventual agent oracle that reports true if at least one agent is present in the network or false otherwise. This system is referred in the following TIP (Tiny Interaction Protocols).

\section{Token Circulation in TIP}

\subsection{Impossibility results for token circula- tion in TIP}

In the following we show the necessity of additional assumptions in order to provide uniform solutions for self-stabilizing leader election or token circulation in TIP (Tiny Interaction Protocols). Notice that memory is an important factor that may help bypassing many of the impossibility results stated below however additional memory means additional corruptions so the system should pay additional time and effort in order to be stabilized. The following note restates in the context of the new interaction model results already known in the classical distributed systems.

Note 1. Let $\mathcal{S}$ be a TIP. It is impossible to guarantee the presence of a unique agent in $\mathcal{S}$ without additional assumptions.

The intuition of the above result is as follows: without additional assumptions it is impossible to decide if the system already has a unique agent, hence new agents may be introduced infinitely and system never converges to a configuration with a unique agent. That is, consider a chain topology and two initial configurations one without any agent, the other with an agent hold by D (see Figure 1). Since the system can start in any configuration both configurations may be initial configurations for a legal execution of the system.

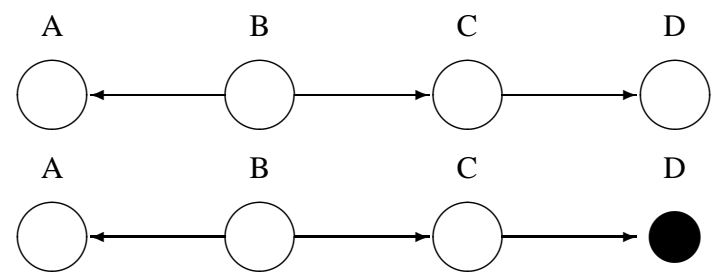

Figure 1. Two initial configurations for Note 1
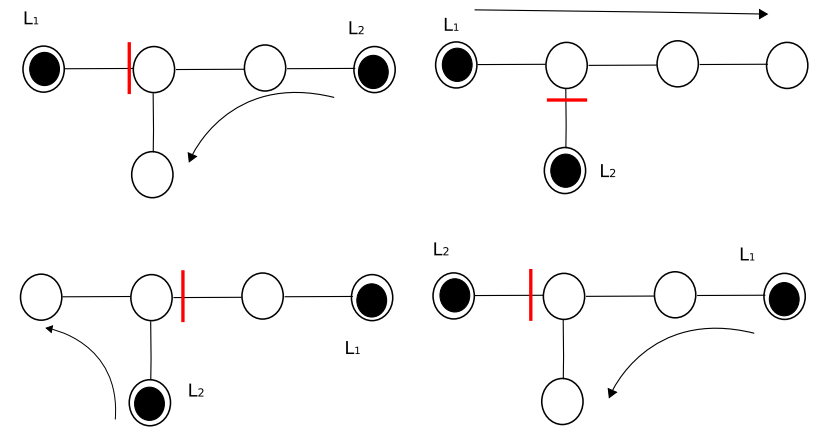

Figure 2. Scheduling for the impossibility result of Lemma 1

Consider node B. It can interact only with nodes A and C. Nodes $\mathrm{A}$ an $\mathrm{C}$ hold no agent in both configurations so in the B's view these two configurations are identical. The following cases can occur:

- B introduces a new agent in the first configuration. Since $\mathrm{B}$ has the same view in both configurations, B will execute the same action in the second configuration as well. This will transform the second configuration in an illegitimate one since it will contain two agents.

- B does not introduce a new agent and no other node becomes agent holder. The first configuration is illegitimate and stays illegitimate for ever.

Hence, we can exhibit infinite executions that never converge to a unique mark legitimate configuration.

Note 2. Note 1 does not hold for a system with two nodes. In this case a simple self-stabilizing algorithm is the following: if neither the initiator nor the responder are agent holders then one of them create an agent; if both the initiator and the responder are agent holders then one of them becomes agent free.

In the following we prove that in TIP with general acyclic graph topology, self-stabilizing unique token circulation is impossible even with the help of $A$ ? and the global fairness assumption.

LEMMA 1. Let $\mathcal{S}$ be a TIP with arbitrary topology. No deterministic self-stabilizing unique agent circulation algorithm exists in $\mathcal{S}$, even with the help of agent detector $A$ ? and the global fairness assumption.

PROOF. Assume a self-stabilizing deterministic unique agent 
circulation algorithm for general acyclic graphs, that works under global fairness with the help of $A$ ?. Consider a graph with two agents $L_{1}$ and $L_{2}$ (see Figure 2). Call the nodes with more than two edges traffic lights. These traffic lights are always red in the direction of one of the two agents, so one agent cannot enter the traffic light node while the other one can cross all the edges of that node but the red one. In such conditions we show that deterministic unique agent circulation is impossible, even under the global fairness assumption. Due to the red light the two agents never interact. Thanks to the red/light, the graph is divided in two parts. By the fairness assumption each agent visits each node of its component. Since the fair scheduler changes the direction of the red/light infinitely many times, each agent visits each node infinitely often without ever interact with the other agent Figure 2 Moreover the agent detector $A$ ? becomes useless because the red light works regardless of its indications. Since the two agents never interact the unique agent circulation behaviour is never verified.

\section{Self-stabilizing Unique Token Circula- tion}

In this section we propose deterministic and probabilistic solutions for token circulation in population protocols with weak agents.

Each node can hold either an agent that will represent the token abstraction or nothing - (following the result of the LocalAgent?() invocation) and each node receives its current input true $(\mathrm{T})$ or false $(\mathrm{F})$ from $A$ ?. $A$ ? returns $(\mathrm{T})$ when at least one agent is present in the network and $(\mathrm{F})$ when no agent is present.

\subsection{Deterministic Unique Token Circula- tion on Chains}

In the following we consider chain topologies and propose self-stabilizing deterministic algorithms for token circulation under global fairness.

Intuitively the algorithm works as follows. A clean node(a node without token) becomes agent holder, when the agent detector signals the absence of any agent in the system (Rule 2). When two nodes holding an agent each interact, the responder becomes clean (Rule 1). If the responder has an agent and the initiator is a clean node, the latter creates an agent and the former becomes clean (Rule 3). Otherwise, no state change occurs. Note that the wild-card symbol, $*$, is used to replace any value.

$$
\begin{aligned}
& \text { Rule 1. }((\boldsymbol{\phi}, *),(\boldsymbol{\phi}, *)) \longrightarrow((\boldsymbol{\phi}),(-)) \\
& \text { Rule 2. }((-, F),(-, *)) \longrightarrow((\boldsymbol{\phi}),(-)) \\
& \text { Rule 3. }((-, *),(\mathbf{\phi}, *)) \longrightarrow((\boldsymbol{\$}),(-))
\end{aligned}
$$

Algorithm 4.1: Unique Token Circulation on chains

LEMMA 2. Let $\mathcal{S}$ be a TIP system. Algorithm 4.1 converges to a legitimate configuration for unique token circulation under asynchronous scheduler and global fairness assumptions.
PROOF. Let $e$ be an execution of Algorithm 4.1 starting in an illegitimate configuration, $c$. The following situations are possible.

- There is no agent in $c$. In this case, all pairs of nodes are enabled for Rule 2 and the scheduler has to chose at least one pair of these nodes. After their execution at least one agent is introduced in the system. Due to the fairness assumption the agent will visit each node of the network.

- There are several agents in $c$. For the sake of simplicity we assume two agents. In a chain topology, Rule 3 and the fairness assumption make each agent visit all nodes. Assume the two agents never meet. This is equivalent to say that there is at least a node that is never visited by an agent which is impossible by the global fairness assumption. When the two tokens become neighbours the execution of Rule 1 reduces the number of agents becomes 1 and the proof reduces to the first case.

LEMma 3. Let $S$ be a TIP system executing Algorithm 4.1. $S$ self-stabilizes to the token circulation specification under an asynchronous scheduler and global fairness assumptions.

Proof. Following Lemma2 $S$ converges to a legitimate configuration in a finite number of steps. By the fairness assumption the unique agent in the network will visit each node infinitely often.

\subsection{Self-stabilizing Token Circulation in Arbitrary Graphs}

In the following we propose a probabilistic self-stabilizing algorithm that solves the unique token circulation using the agent detector $A$ ?. The algorithm works under k-bounded scheduler. The algorithm idea is as follows. Agents perform random walks in order to find and destroy other agents. If no agent is reported by $A$ ? then new agents are introduced in the system.

A clean node creates an agent when there is no agent in the system $A$ ? $=\mathrm{F}$ (Rule 2). If two agent holders interact (one of them as initiator and the other as responder), the responder looses its agent (Rule 1). If an agent holder interacts with a clean node, the agent is moved from the initiator to the responder with a probability of $1 / 2$ (Rule 3 ) and if the initiator is clean and the responder has an agent, then the agent moves with probability $1 / 2$ from the latter to the former. Rule 2 introduces agents when $A$ ? reports their absence. Rule 1 destroys extra agents. Rule 3 allows agents to travel in the network in order to meet each other and eventually be destroyed via Rule 1 .

LEMMA 4. An agent covers infinitely often a virtual ring that includes all nodes in the system.

PROOF. Assume there is a node of the graph that is never visited by the agent. Either the agent is blocked in a node or the agent cycles in a part of the graph. In the first case the agent holder is enabled for the Rule 3. The probability for this node 
In the following we discuss the leader election feasibility in TIP. We increase the power of the system by adding an eventual agent detector.

Rule 1. $((\boldsymbol{\phi}, *),(\boldsymbol{\phi}, *)) \longrightarrow((\boldsymbol{\phi}),(-))$

Rule 2. $((-, F),(-, *)) \longrightarrow((\boldsymbol{A}),(-))$

Rule 3. $((\boldsymbol{\phi}, *),(-, *)) \longrightarrow \begin{cases}\operatorname{Pr}(1 / 2) & ((-),(\mathbf{\phi})) \\ \operatorname{Pr}(1 / 2) & ((\mathbf{\phi}),(-))\end{cases}$

Algorithm 4.2: Probabilistic agent circulation

to keep the agent infinitely is $0: \lim _{s \rightarrow \infty}\left[\left(\frac{1}{2}\right)^{S}\right]$. In the second case, either the agent is pushed back and forth between two nodes or the agent travels in a cycle. Both cases are impossible due to the fairness assumption.

COROLlary 1. Two agents that cover two virtual rings visit at least one common node.

LEMMA 5. Let e be an execution of Algorithm 4.2 starting in a configuration with two agents. Eventually, two agent holders interact under the k-bounded scheduler assumptions.

Due to space limitation the proof of the above lemma is not provided. The proof uses similar arguments as the correctness of classical self-stabilizing token based schemes [15].

LEMMA 6. Let e be an execution of Algorithm 4.2 starting in an arbitrary configuration. e converges to a legitimate configuration.

Proof. Suppose there are no tokens in the initial configuration of $e$. So from some point on, every node receives false from $A$ ?. By Rule 2 the initiators declare themselves agent holders and the system reaches a configuration with one ore more agents. Starting from this configuration, some clean nodes may receive false from their detector and continue to inject agents but there is a point in the execution from which $A$ ? returns true to every node in the system. From this point onward no new agents are injected in the system. Suppose the system in a configuration with more than two agents and $A$ ? returns true to every node in the system. Let $k$ be the number of agents in this configuration. By Lemma 5 two agents in this set eventually interact and by Rule 1 one of them disappears. So starting from a configuration with $k$ agents in a finite number of steps the number of agents drops to $k-1$. The process is iterated until the system reaches a legitimate configuration.

The correctness of our system is a direct consequence of the previous lemmas.

THEOREM 1. The system executing Algorithm 4.2 verifies the token circulation specification assuming a bounded scheduler.

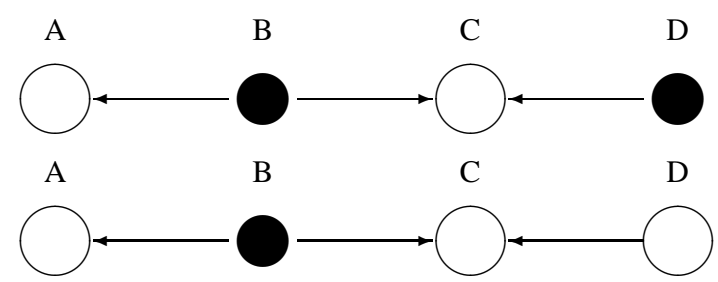

Figure 3. Two initial configurations for Lemma 7

\section{Global and Local Leader Election in TIP}

In the following we discuss the leader election feasibility in TIP. We increase the power of the system by adding an eventual agent detector. We address both the global leader election and its local version.

\subsection{Impossibility results related to leader election in TIP}

LEMMA 7. Let $\mathcal{S}$ be a TIP. There is no deterministic or probabilistic uniform self-stabilizing silent leader-election algorithm in $S$ even with the help of an eventual agent detector A? without additional assumptions.

PROOF. Intuitively the proof goes as follows. Suppose the presence of two leaders (each leader holds an agent) and none of them can notice the existence of the other one. Even with the help of an agent detector $A$ ?, for each of them it is impossible to decide if it is the only leader or there is another leader in the system.

Consider two configurations, $c$ and $c_{1}$, and a chain topology: one with two leaders in nodes B and D and the other one with a leader in node B (see Figure 3). Note that $c_{1}$ is a terminal configuration since it is legitimate.

Node $\mathrm{B}$ has visibility only on its neighbors and $A$ ? can notify only if there is at least an agent in the network, so from its point of view, the two configurations are identical. The following cases arise:

- B holds its agents. Since B has the same view in both configurations, the first configuration is still illegitimate since it has two leaders.

- B becomes agent free. Since B has the same view in both configuration, the same action is executed in both configurations. Two new configurations are obtained: $c^{\prime}$ and $c_{1}^{\prime}$ and in $c_{1}^{\prime}$ there is no leader. If in $c_{1}^{\prime}$, B and $\mathrm{D}$ decide to become leader, since to both of them $\Omega$ ? returns false, the system returns to a configuration similar to the initial configuration and the system is not any more silent.

- B pushes the leader mark to one of its neighbors (say C) and C may do the same since it has the same "view" as $B$ in the previous configuration. So, the leader mark arrives on $D$ which has the same view in both the configurations (the legitimate and illegitimate configuration). 
Overall, even helped by $\Omega$ ? it's impossible to assert if the leader-election configuration is reached or not without additional assumptions.

LEMMA 8. Let $\mathcal{S}$ be a TIP with ring topology of odd size. There is no deterministic or probabilistic uniform selfstabilizing silent local leader-election algorithm in $\mathcal{S}$ without additional assumptions.

Proof. Consider a ring topology of odd size and the following initial configuration $(n, n, a, n, a, \ldots, a)$ where $n$ denotes an empty node and $a$ denotes an agent holder. Whatever the fair scheduling the configuration cannot stabilize to a configuration where clean node alternate with agent nodes since the size of the ring is odd.

In order to bypass the impossibility result for the case of odd size rings we add a k-distance agent detector, $A^{k}$ ?. Differently from the global agent detector the k-distance agent detector reports if up to distance $k$ there is an agent. Algorithm 5.1 implements local leader election in general graphs using $A^{1}$ ? The algorithm idea is the same as for the even sized rings. The main difference comes in the interaction of clean nodes. They introduce a new agent only and only if they have no agent in their neighbourhood. Each node execution the algorithm has either an agent agent (the LocalAgent?() returns true) or is empty and receives the input of $A^{1}$ ?

Rule 1. $((\boldsymbol{\phi}, *),(\boldsymbol{\phi}, *)) \longrightarrow((\boldsymbol{\phi}, *),(-, *))$

Rule 2. $((-, F),(-, *)) \longrightarrow((\boldsymbol{\phi}, *),(-, *))$

Algorithm 5.1: Local leader election for even sized rings

LEMMA 9. Algorithm 5.1 is a silent implementation of local leader election in general graphs under asynchronous scheduler and global fairness assumption.

Proof. Intuitively, the proof goes as follows. Let $T$ be the set of conflicting pair of neighbors. That is, either both have an agent or they are clear and have no neighbors with an agent. Due to the fairness assumption, each of these pair of nodes will eventually interact and apply either Rule 1 or Rule 2. After each interaction the size of $T$ eventually decreases. In a finite number of interactions the systems stabilizes.

\section{Conclusions and discussions}

In this paper we focused on the self-stabilizing token circulation and (local) leader election solutions in population protocol model augmented with agents and the eventual agent detector. The eventual agent detector eventually reports the presence or the absence of an agent. We considered a very weak model of agents and nodes: anonymous, uniform and oblivious. Agents have no memory while nodes in the population have only one Boolean slot (not persistent). In this model we proposed deterministic silent solutions for self-stabilizing local leader election. Moreover, we addressed the token circulation problem. Note that the agent paradigm materializes the token abstraction. We proposed deterministic and probabilistic solutions and proved the necessity of the eventual agent detector even in environments helped by randomization.
The proposed model unifies several models for distributed interactions: population protocols, robots with global and local visibility (via the oracle paradigm) and the agents paradigm. Therefore, the current work opens several research directions. An interesting open issue would be the study of the power of this model when agents and/or nodes have local memory. Furthermore, an another interesting issue is to be explored the impact on the population stabilization of the full powered agents that execute some code when guested by a node.

\section{References}

[1] D. Angluin, J. Aspnes, Z. Diamadi, M. J. Fischer, and R. Peralta, "Computation in networks of passively mobile finite-state sensors," Distributed Computing, pp. 235-253, Mar. 2006.

[2] D. Angluin, J. Aspnes, M. J. Fischer, and H. Jiang, "Selfstabilizing population protocols," TAAS, vol. 3, no. 4, 2008.

[3] D. Angluin, J. Aspnes, and D. Eisenstat, "Fast computation by population protocols with a leader," Distributed Computing, vol. 21, no. 3, pp. 183-199, 2008.

[4] C. Delporte-Gallet, H. Fauconnier, R. Guerraoui, and E. Ruppert, "When birds die: Making population protocols fault-tolerant," in DCOSS, 2006, pp. 51-66.

[5] - "Secretive birds: Privacy in population protocols," in OPODIS, 2007, pp. 329-342.

[6] M. Fischer and H. Jiang, "Self-stabilizing leader election in networks of finite-state anonymous agents." in OPODIS, 2006, pp. 395-409.

[7] S. Cai, T. Izumi, and K. Wada, "Space complexity of self-stabilizing leader election in passively-mobile anonymous agents," in SIROCCO, 2009, pp. 113-125.

[8] J. Beauquier, J. Burman, and S. Kutten, "Making population protocols self-stabilizing," in SSS, 2009, pp. 90-104.

[9] S. Ghosh, "Agents, distributed algorithms, and stabilization," in COCOON, 2000, pp. 242-251.

[10] J. Beauquier, T. Hérault, and E. Schiller, "Easy stabilization with an agent," in WSS, 2001, pp. 35-50.

[11] S. Dolev, Self-Stabilization. MIT Press, 2000.

[12] T. D. Chandra, V. Hadzilacos, and S. Toueg, "The weakest failure detector for solving consensus," J. ACM, vol. 43, no. 4, pp. 685-722, 1996.

[13] P. Flocchini, D. Ilcinkas, A. Pelc, and N. Santoro, "Remembering without memory: Tree exploration by asynchronous oblivious robots," Theor. Comput. Sci., vol. 411, no. 14-15, 2010.

[14] C. Lenzen, J. Suomela, and R. Wattenhofer, "Local Algorithms: Self-Stabilization on Speed," in 11th International Symposium on Stabilization, Safety, and Security of Distributed Systems (SSS), Lyon, France, November 2009.

[15] J. Beauquier, M. Gradinariu, and C. Johnen, "Randomized self-stabilizing and space optimal leader election 
under arbitrary scheduler on rings," Distributed Computing, vol. 20, no. 1, pp. 75-93, 2007.

\section{Annexes}

\subsection{Local leader election for even-sized rings}

Intuitively the algorithm works as follows. A non-leader becomes a leader when the responder is not a leader (Rule 2). When two local leaders interact, the initiator becomes nonleader (Rule 1). Each node can hold either an agent, $\mathbf{\phi}$, or - .

Rule 1. $((\mathbf{\$}),(\boldsymbol{\$})) \longrightarrow((\mathbf{\$}),(-))$

Rule 2. $((-),(-)) \longrightarrow((\mathbf{\$}),(-))$

Algorithm 7.1: Local leader election for even sized rings

Definition 6. A configuration of Algorithm 7.1 is legitimate if in each neighbourhood there is only one process holding an agent. This process will be called the local leader.

LEMMA 10. Algorithm 7.1 is a silent implementation of local leader election in even-sized rings using global fairness.

PROOF. Intuitively, the proof goes as follows. Eventually, two neighbors interact and one of them becomes leader. Since the size of the ring is even the following cases can happen. Either all nodes hold an agent or all of them are clean. In one round of interaction the system converges to a legitimate configuration (either applying Rule 1 or Rule 2). All the other cases reduce to the above case. 